\title{
Applications and Manipulation Method of Microbeads in Biological Field
}

\author{
Yapeng Yuan ${ }^{1}$, Yaxiaer Yalikun ${ }^{2,3}$ and Yo Tanaka*2 \\ ${ }^{1}$ Yuan lab, China \\ ${ }^{2}$ Center for Biosystems Dynamics Research, RIKEN, Japan \\ ${ }^{3}$ Division of Materials Science, Nara Institute of Science and Technology, Japan
}

Received: 制: September 05, 2018; Published: 制 September 17, 2018

*Corresponding author: Yo Tanaka, Center for Biosystems Dynamics Research, RIKEN, Japan

Yaxiaer Yalikun, Division of materials Science, Nara Institute of Science and Technology, Nara, Japan

\begin{abstract}
In the fields of cell biology, such as biomolecular chemistry and genomics, microbeads play an important role as a powerful analysis tool. To realize above applications, manipulation of size selection of microbeads is required. Therefore, in this paper we summarized newest manipulation methods for size selection of microbeads and their applications. We believe our paper offers specific reference for those researchers intend to use microbeads in biological field.
\end{abstract}

Keywords: Microbeads; Manipulation; Application; Size Selection

\section{Introduction}

Currently, due to their availability in different aspects [1-4] microbeads are widely used for a variety of different laboratory procedures and protocols. Microbeads technology has great effect on biological assay in molecular and genomic researches [5,6], for example, bar-coded (encoded) microbeads have been used in beadbased arrays [7]. Also, microbeads are cost saving and have excellent mechanical and thermal stability. Microbeads can be coated with an assay specific reagent, thereby facilitating high-throughput affinitybased capturing and detection of target biological molecules from a small sample volume [8-10]. In addition, microbeads offer advantages when used to perform chemical tests in microfluidic chips: they could be introduced into the device at any time, can be easily functionalized off-chip with specific receptors for analytes, can have a very large surface area, enable absorption, reaction, and electrochemical assay formats, and can be packed for washing and signal reading steps [11]. Improved methods of tagging and handling microbeads have also allowed commercial products such as Luminex bead array technology to be used in applications of diagnostics and drug discovery [12]. Microbeads can also provide a suitable three-dimensional environment for cell growth and differentiation [13]. Therefore, separation and manipulation of microbeads has great importance in medical diagnostics, immunology, chemical and biological analyses. For example, microbead sorting and patterning technology would allow direct identification and mapping of analyte binding to size specific microbeads which are encoded with different target reagents
$[7,14,15]$. Moreover, microbeads with an added physical dimension such as bead sizes, can be utilized for detecting cytokines and simultaneously measuring multiple analytes for immunoassay [16]. Hence, this paper briefly reviews recent advanced methods of manipulating and handling microbeads and their applications.

\section{Methods of Microbeads Manipulation and its Application}

Microfiltration: In general, microfiltration is recognized as one of the most widely used techniques in the separation of microbeads, as it depends on the size of microbeads. The basic method of microfiltration is to use the conventional membrane-based microfilter. By employing membranes with different pore sizes, multicomponent particle filtration can be achieved. With suitable pore size, this method can theoretically separate microbeads of any size. To increase the capture efficiency of smaller cells or particles, Sun et al. [17] developed a strategy employing modified microbeads to specifically bind onto target cells, which enlarged the size of target cells. Lin et al. [18] focused on the size amplification of cells by tagging them with $3 \mu \mathrm{m}$ microbeads. In addition, Wong et al. [19] designed a 3D micro-traps array to filter out smaller diameter beads while retaining larger ones, which would be useful in applications like bead microarray assays.

Inertia and Dean Flow Separation: Recently, inertial separation plays a significant role in the manipulation of microbeads, due to the feature of single-step, highly-selective [20]. Microbeads of different sizes achieve different lateral location inside the 
channel due to the balance of inertial lift force and dean drag force [21]. This method is capable of manipulating the microbeads more than $1 \mu \mathrm{m}$ of diameter. Yoon et al. [22] reported a microscale benefit of a secondary flow obtained in a curved rectangular microchannel and achieved the separation and sorting of microbeads by their size using secondary flow. Wang et al. [23] exhibited a novel inertial microfluidic sorter that was compatible with the roll-to-roll hot embossing process for size-based sorting of microbeads. With optimized flow conditions and channel dimensions, the sorting efficiency of a mixture of $15 \mu \mathrm{m}$ and $10 \mu \mathrm{m}$ diameter microbeads was more than $97 \%$.

Dielectrophoresis: Manipulation of microbeads can be achieved by using dielectrophoresis (DEP) and compact electrode layouts [24]. The magnitude and direction of the displacement caused by the DEP force are depended on the electric field and the feature of microbeads or solutions. Jaione et al. [25] presented an efficient and compact lateral displacement separation method for microbeads based on negative DEP forces employing a capillarydriven microfluidic chip and an optimized electrode layout, which achieved the efficient separation of $10 \mu \mathrm{m}$ and $5 \mu \mathrm{m}$ beads, with $98 \%$ of all concentrated beads sorted in two separate streams. Jaione's group also presented a highly efficient and reversible mechanism for manipulating microbeads by combining dielectrophoresis (DEP) with mechanical traps [26]. Khashayar et al. [27] designed a DEP system that utilized curved microelectrodes integrated into microfluidic system and applied microbeads of 1, 6 and $15 \mu \mathrm{m}$ to the system and their response to the DEP field was studied at different frequencies of 100,200 , and $20 \mathrm{MHz}$. Finally, the separation of microbeads of different sizes was successfully achieved.

Magnetic Separation: Magnetic manipulation is a common technique used to obtain a highly pure population of microparticles/beads or cells from a mixed solution [28]. In biological analysis, to obtain the target cells, tag the cells with magnetic microbead conjugated antibodies and then place the mixed solution in a magnetic field. Under the action of magnetic field, the labeled cells with magnetic microbead are separated from the mixed solution [29]. Tomin et al. [30] has developed lectin-conjugated magnetic microbeads recognizing specific glycans of apoptotic cells and its use for detection and separation of dying cells. In addition, depending of the size of the magnetic microbeads, the behavior of different size microbeads in the magnetic field is different. Effect of the field is large on larger particle than on the smaller one, so the larger microbeads present in the sample are separated from the smaller microbeads [31].

Acoustic Separation: Acoustic-based method has emerged as a new and ideal technology for use in microbeads separation, and many applications of acoustic manipulation have been developed [32]. The acoustic radiation force acting on the microbeads is depended on the property of the microbeads, and one property that can be exploited for the separation of microbeads based on size [33]. Jonathan et al. [34] developed a tunable acoustic separation device which could sort microbeads or cells based on a range of sizes, and the device was capable of sorting an arbitrary range of mi- crobead sizes between 3 and $10 \mu \mathrm{m}$ in diameter with high efficiency (98\%). Jeonghun et al. [35] presented a method that used a standing surface acoustic wave to continuously separate microbeads in a size-gradient manner in a microchannel flow, and three microbeads with different diameters $(1,5$ and $10 \mu \mathrm{m})$ were successfully separated with high efficiency.

Microfiltration and Inertia and dean flow separation have simpler structure and do not employ external fields for the operation, but microfiltration method requires complicated fabrication procedure to assemble the device with filters and the inertial separation requires sophisticated channels that facilitate movement of the microbeads. DEP, magnetic or acoustic separation use various forms of external fields to generate external force to manipulate and separate particles with relatively better performance and shorter time.

\section{Conclusion}

In this paper, a brief review of methods of microbeads manipulation and their applications is discussed. Each method has its own characteristics and advantages. In the future, devices would make a combination of these methods to develop more suitable separation and manipulation techniques.

\section{Acknowledgment}

This work was supported by JSPS KAKENHI grant no. 15K11918 and TEPCO Memorial Foundation, Japan.

\section{References}

1. Leslie SK, Nicolini AM, Sundaresan G, Zweit J, Boyan BD, et al. (2016) Development of a cell delivery system using alginate microbeads for tissue regeneration. Journal of Materials Chemistry B 4: 3515-3525.

2. Basan H, Dinc M, Mizaikoff B (2018) Inhibitor-assisted synthesis of molecularly imprinted microbeads for protein recognition. Analytical Methods 9: 997-1005.

3. Foti L, Sionek A, Stori EM, Soares PP, Pereira MM, et al. (2015) Electrospray induced surface activation of polystyrene microbeads for diagnostic applications. Journal of Materials Chemistry B 13: 2725-2731.

4. Labriola NR, Mathiowitz E, Darling EM (2017) Fabricating polyacrylamide microbeads by inverse emulsification to mimic the size and elasticity of living cells. Biomaterials Science 5(1): 41-45.

5. Zeng H, Inoue Y, Moritani K, Nishiwaki M, Nakajima H, et al. (2012) Controllable construction of ordered three-dimensional microbeads structure and its application in enzyme-linked immunosorbent microarray. Sensors and Actuators B Chemical 168: 446-452.

6. Manesse M, Phillips AF, Lafratta CN, Palacios MA, Hayman RB, Walt DR (2013) Dynamic microbead arrays for biosensing applications. Lab on a Chip 13(11): 2153-2160.

7. Jun BH, Kang H, Lee YS, Jeong DH (2012) Fluorescence-based multiplex protein detection using optically encoded microbeads. Molecules 17(3): 2474-2490.

8. Omer AM, Tamer TM, Hassan MA, Rychter P, Mohy Eldin MS (2016) Development of amphoteric alginate/aminated chitosan coated microbeads for oral protein delivery. International Journal of Biological Macromolecules 92: 362-370.

9. Lu B, He Q, He Y, Chen X, Feng G, et al. (2018) Dual-channel-coded microbeads for multiplexed detection of biomolecules using assembling of quantum dots and element coding nanoparticles. Analytica Chimica Acta 1024: 153-160. 
10. Braeckmans K, De Smedt SC, Leblans M, Pauwels R, Demeester J (2002) Encoding microcarriers: present and future technologies. Nature Reviews Drug Discovery 1: 447-456.

11. Gong X, Yan H, Yang J, Wu Y, Zhang J, et al. (2016) High-performance fluorescence-encoded magnetic microbeads as microfluidic protein chip supports for AFP detection. Analytica Chimica Acta 939: 84-92.

12. Prabhakar U, Eirikis E, Davis HM (2002) Simultaneous quantification of proinflammatory cytokines in human plasma using the LabMAPTM assay. Journal of Immunological Methods 260(1): 207-218.

13. Kang SW, Yang HS, Seo SW, Han DK, Kim BS (2008) Apatite-coated poly(lactic-co-glycolic acid) microspheres as an injectable scaffold for bone tissue engineering. Journal of Biomedical Materials Research A 85(3): 747-756.

14. Gong X, Zhang Q Cui Y, Zhu S, Su W, et al. (2013) A facile method to prepare high-performance magnetic and fluorescent bifunctional nanocomposites and their preliminary application in biomolecule detection. Journal of Materials Chemistry B: 15

15. Wilson R, Spiller DG, Prior I A, Veltkamp KJ, Hutchinson A (2007) A Simple Method for Preparing Multiplexed Detection. Acs Nano 1(5): 487-293.

16. Tsai SW, Chang YH, Yu JL, Hsu HW, Rau LR, et al. (2016) Preparation of nanofibrous structure of mesoporous bioactive glass microbeads for biomedical applications. Materials 9(6): 487.

17. Sun N, Li X, Jaione Jaione Z, Li Y, Pei R (2018) High-purity capture of CTCs based on micro-beads enhanced isolation by size of epithelial tumor cells (ISET) method. Biosensors and Bioelectronics 102: 157-263.

18. Lin MX, Hyun KA, Moon HS, Sim TS, Lee JG, et al. (2013) Continuous labeling of circulating tumor cells with microbeads using a vortex micromixer for highly selective isolation. Biosensors and Bioelectronics 40(1): 63-67.

19. Wong CC, Liu Y, Wang KY, Rahman ARA (2013) Size based sorting and patterning of microbeads by evaporation driven flow in a 3D micro-traps array. Lab on a Chip18: 3663-3667.

20. Rothbauer M, Zirath H, Ertl P (2018) Recent advances in microfluidic technologies for cell-to-cell interaction studies. Lab on a Chip 18(2): 249-270.

21. Gossett DR, Di Carlo D (2009) Particle focusing mechanisms in curving confined flows. Analytical Chemistry 81(20): 8459-8465.

22. Yoon DH, Ha JB, Bahk YK, Arakawa T, Shoji S, et al. (2009) Size-selective separation of micro beads by utilizing secondary flow in a curved rectangular microchannel. Lab on a Chip 9: 87-90.

\section{ISSN: 2574-1241}

DOI: 10.26717/BJSTR.2018.09.001742

Yo Tanaka. Biomed J Sci \& Tech Res

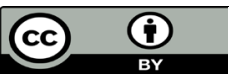

This work is licensed under Creative Commons Attribution 4.0 License

Submission Link: https://biomedres.us/submit-manuscript.php
23. Wang X, Liedert C, Liedert R, Papautsky I (2016) A disposable, roll-toroll hot-embossed inertial microfluidic device for size-based sorting of microbeads and cells. Lab on a Chip 16(10):1821-1830.

24. Sancho M, Martínez G, Muñoz S, Sebastián JL, Pethig R (2010) Interaction between cells in dielectrophoresis and electrorotation experiments. Biomicrofluidics 4(2): 022802.

25. Tirapu Azpiroz J, Temiz Y, Delamarche E (2017) Dielectrophoretic microbead sorting using modular electrode design and capillary-driven microfluidics. Biomedical Microdevices 19(4): 1-8.

26. Tirapu Azpiroz J, Temiz Y, Delamarche E (2015) Arraying single microbeads in microchannels using dielectrophoresis-assisted mechanical traps. Applied Physics Letters 107(20): 1-5.

27. Khoshmanesh K, Zhang C, Nahavandi S, Tovar Lopez FJ, Baratchi S, et al. (2010) Size based separation of microparticles using a dielectrophoretic activated system. Journal of Applied Physics 108(3).

28. Hejazian M, Li W, Nguyen NT (2015) Lab on a chip for continuous-flow magnetic cell separation. Lab on a Chip 15(4): 959-970.

29. Bhattacharjee J, Das B, Mishra A, Sahay P, Upadhyay P (2017) Monocytes isolated by positive and negative magnetic sorting techniques show different molecular characteristics and immunophenotypic behaviour. F1000Research 6: 2045.

30. Tomin A, Dumych T, Kril I, Antonyuk V, Chopyak V, et al. (2016) Magnetic separation of apoptotic cells with lectin-conjugated microparticles: Magnetische Abtrennung apoptotischer Zellen mit Lektin-konjugierten Mikropartikeln. Materialwissenschaft Und Werkstofftechnik 47: 189192.

31. Lenshof A, Laurell T (2010) Continuous separation of cells and particles in microfluidic systems. Chemical Society Reviews 39: 1203-1217.

32. Guldiken R, Jo MC, Gallant ND, Demirci U, Zhe J (2012) Sheathless sizebased acoustic particle separation. Sensors 12(1): 905-922.

33. Liu Y, Lim KM (2011) Particle separation in microfluidics using a switching ultrasonic field. Lab on a Chip 11(18): 316-373.

34. Adams JD, Soh HT (2010) Tunable acoustophoretic band-pass particle sorter. Applied Physics Letters 97(6): 1-4.

35. Nam J, Lee Y, Shin S (2011) Size-dependent microparticles separation through standing surface acoustic waves. Microfluidics and Nanofluidics 11(3): 317-326. 